\title{
Isospectral membranes: a connection between shape and density
}

\author{
Ian W Knowles ${ }^{1}$ and Maeve L McCarthy ${ }^{2}$ \\ ${ }^{1}$ Department of Mathematics, University of Alabama at Birmingham, 452 Campbell Hall, \\ 1300 University Boulevard, Birmingham, AL 35294-1170, USA \\ ${ }^{2}$ Department of Mathematics \& Statistics, Murray State University, 6C Faculty Hall, Murray, \\ KY 42071-3341, USA \\ E-mail: maeve.mccarthy@murraystate.edu
}

Received 13 April 2004, in final form 18 June 2004

Published 4 August 2004

Online at stacks.iop.org/JPhysA/37/8103

doi:10.1088/0305-4470/37/33/009

\begin{abstract}
A connection is established via conformal maps between vibrating membranes that are isospectral with respect to shape and those that are isospectral with respect to density. In particular, inhomogeneous circular membranes are constructed that are isospectral to polygonal membranes of uniform density via the Schwarz-Christoffel mapping. Although some corners of the polygons lead to singularities in the constructed densities, the densities are shown to be integrable.
\end{abstract}

PACS numbers: $02.30 . F n, 02.30 . J r, 02.60 . \mathrm{Lj}$

Mathematics Subject Classification: 30C30, 30E25, 35J55, 35P99, 35R30, $74 \mathrm{~K} 15$

(Some figures in this article are in colour only in the electronic version)

\section{Introduction}

The vibrating membrane has many applications including drums, microphones, loudspeakers [16] and transducers [17]. A variety of techniques for the solution of the associated boundary value problem have been studied [14]. We use one of these techniques, conformal mappings, here to investigate the connection between two classes of isospectral membranes, i.e. membranes whose eigenfrequency spectra are identical. Conformal mappings have often been used to solve boundary value problems arising in applications. The basic premise is that the transformed problem is posed over a nicer geometry, thereby making the problem more tractable. This arises in the study of vibrating membranes [14], electromagnetism [2] and fluid flow [15]. Although conformal mappings are generally used only to change the geometry of a problem, they can also be used to change the coefficients of a problem. Here we use a 

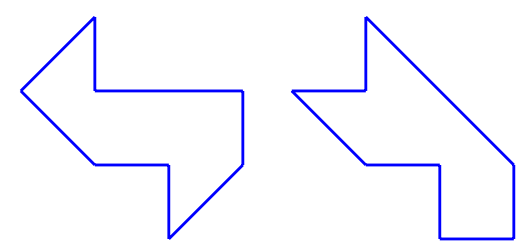

Figure 1. Isospectral shapes found by Gordon, Webb and Wolpert.
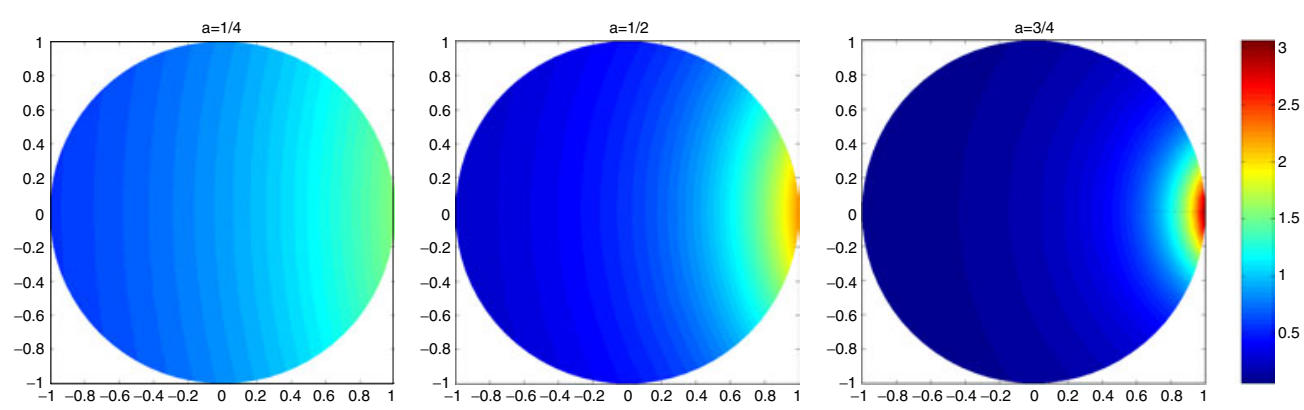

Figure 2. Densities isospectral to a uniform circular membrane found by Gottlieb for $a=$ $1 / 4,1 / 2,3 / 4$.

conformal map to study the connection between membranes that are isospectral with respect to shape and those that are isospectral with respect to density.

In the context of the homogeneous vibrating membrane, the existence of isospectral shapes allows us to answer 'no' to the famous question 'Can one hear the shape of a drum?' [12]. Such shapes were first constructed for the two-dimensional membrane by Gordon, Webb and Wolpert [4, 3, 9] using a result of Sunada [22]. The examples constructed included the polygons shown in figure 1 .

Gottlieb [10] used a conformal map to construct a class of inhomogeneous unit circular membranes with density $\rho(x, y)$ that were isospectral to a homogeneous unit circular membrane with constant density $\rho_{0}$ :

$$
\rho(x, y)=\frac{\rho_{0}\left(1-a^{2}\right)^{2}}{\left[(1-a x)^{2}+a^{2} y^{2}\right]^{2}}
$$

for each $a \in(0,1)$. Figure 2 shows examples of the Gottlieb isospectral densities. Recently, Gottlieb [11] generalized his previous result and established a similar construction showing the isospectrality of two inhomogeneous unit circular membranes with densities $\rho_{1}(x, y)$ and $\rho_{2}(x, y)$ respectively:

$$
\rho_{2}(x, y)=\frac{\rho_{1}(\bar{x}, \bar{y})\left(1-a^{2}\right)^{2}}{\left[(1-a x)^{2}+a^{2} y^{2}\right]^{2}}
$$

for each $a \in(0,1)$, with

$$
\bar{x}=\frac{(x-a)(1-a x)-a y^{2}}{(1-a x)^{2}+a^{2} y^{2}}, \quad \bar{y}=\frac{\left(1-a^{2}\right) y}{(1-a x)^{2}+a^{2} y^{2}} .
$$




\section{Conformal mappings of inhomogeneous membranes}

The technique used is as follows. Let $\Omega$ and $\mathcal{O}$ be domains in the complex planes $z=x+\mathrm{i} y$ and $w=u+\mathrm{i} v$. Let $w=F(z)$ be a one-to-one analytic mapping from $\Omega$ to $\mathcal{O}$. For $\mathcal{O}$ and $\Omega$ strict simply-connected subsets of the complex plane, the existence of the mapping $F$ follows by the Riemann mapping theorem (see e.g. [19, theorem 14.8]). The inhomogeneous clamped membrane with areal density distribution $\rho_{1}(x, y)$

$$
-\Delta U=\lambda \rho_{1}(z) U \quad \text { in } \Omega \quad U=0 \quad \text { on } \partial \Omega
$$

is mapped to the inhomogeneous clamped membrane with areal density distribution $\rho_{2}(u, v)$

$$
-\Delta V=\lambda \rho_{2}(w) V \quad \text { in } \mathcal{O} \quad V=0 \quad \text { on } \partial \mathcal{O}
$$

with

$$
\rho_{1}(z)=\rho_{2}(w)\left|F^{\prime}(z)\right|^{2},
$$

cf Saff and Snider [20].

Since we know that there are isospectral polygonal membranes with uniform density and isospectral circular membranes with varying densities, we will use a conformal map between polygons and circles to construct a new class of isospectral densities for circular membranes that are also isospectral to polygonal membranes.

\subsection{Schwarz-Christoffel mapping}

The Schwarz-Christoffel mapping is the conformal mapping $w=F(z)$ that maps the unit disc $\mathcal{D}=\{z:|z|<1\}$ to a polygon $\mathcal{P}$. If the polygon has $n$ vertices $w_{k}$, given in counter-clockwise order, and interior angles $\alpha_{k} \pi(k=1, \ldots, n)$, then the map has the form

$$
F(z)=A \int^{z} \prod_{k=1}^{n}\left(1-\frac{z}{z_{k}}\right)^{-\beta_{k}} \mathrm{~d} z+B
$$

where $A$ and $B$ are complex constants, $\beta_{k}=1-\alpha_{k}$ and $z_{k}$ are points on the unit circle known as prevertices satisfying $w_{k}=F\left(z_{k}\right)$, cf Ahlfors [1], Driscoll and Trefethen [8]. For a bounded polygon with no cusps or slits, $\alpha_{k} \in(0,2)$. A cusp at the vertex $w_{k}$ yields $\alpha_{k}=0$, while a slit yields $\alpha_{k}=2$. Vertices at infinity yield $\alpha_{k} \in[-2,0]$. In order for the polygon to be closed, $\sum \beta_{k}=2$ and hence $\sum \alpha_{k}=n-2$, where $n$ is the number of vertices. For polygons with $n>3$ the prevertices $z_{k}$ cannot generally be computed explicitly. Their computation is the subject of a great deal of literature, notably [23, 21, 6, 24].

\subsection{Density of a circular membrane resulting from a transformed polygon}

Consider a homogeneous membrane on the polygon $\mathcal{P}$ with constant density, $\rho_{0}$. This polygon can be mapped to the unit disc $\mathcal{D}$ using the inverse of the Schwarz-Christoffel mapping. The resulting circular membrane is inhomogeneous and has density $\rho$, with

$$
\rho(z)=\rho_{0}\left|F^{\prime}(z)\right|^{2}=\rho_{0}|A|^{2} \prod_{k=1}^{n}\left|1-\frac{z}{z_{k}}\right|^{-2 \beta_{k}} .
$$

Clearly, if $\beta_{k}>0$ then $\rho(z)$ has singularities on the boundary of the circular membrane. In that case, $\rho(z)$ is bounded except at $z=z_{k}$ for $k=1, \ldots, n$, and so

$\int_{\mathcal{D}} \rho(z) \mathrm{d} z<\infty \quad \Longleftrightarrow \quad \int_{B_{\varepsilon}\left(z_{k}\right)} \rho(z) \mathrm{d} A<\infty \quad$ for $\quad k=1, \ldots, n$. 
Since there are constants $C_{1}, C_{2}$ such that

$$
C_{1}\left|z-z_{k}\right|^{-2 \beta_{k}} \leqslant \rho(z) \leqslant C_{2}\left|z-z_{k}\right|^{-2 \beta_{k}}
$$

on the ball $B_{\varepsilon}\left(z_{k}\right)$, it suffices to consider

$$
\begin{aligned}
\int_{B_{\varepsilon}\left(z_{k}\right)}\left|z-z_{k}\right|^{-2 \beta_{k}} \mathrm{~d} A & =\int_{B_{\varepsilon}\left(z_{k}\right)} \frac{1}{\left|z-z_{k}\right|^{2 \beta_{k}}} \mathrm{~d} A \\
& =\int_{B_{\varepsilon}(0)} \frac{1}{|z|^{2 \beta_{k}}} \mathrm{~d} A \\
& =\int_{0}^{2 \pi} \int_{0}^{\varepsilon} \frac{1}{r^{2 \beta_{k}}} r \mathrm{~d} r \mathrm{~d} \theta \\
& =2 \pi \int_{0}^{\varepsilon} r^{1-2 \beta_{k}} \mathrm{~d} r \\
& =\left.\frac{\pi r^{2\left(1-\beta_{k}\right)}}{1-\beta_{k}}\right|_{0} ^{\varepsilon}=\left.\frac{\pi r^{2 \alpha_{k}}}{\alpha_{k}}\right|_{0} ^{\varepsilon} .
\end{aligned}
$$

Hence

$$
\int_{\mathcal{D}} \rho(z) \mathrm{d} A<\infty \quad \Longleftrightarrow \quad \alpha_{k}>0 \quad \text { for } \quad k=1, \ldots, n .
$$

In geometric terms, this means that the unit disc density associated with a particular polygon will be integrable provided that the interior angles of the polygon $\alpha_{k} \pi$ are positive. Thus a polygon may have no cusps and must be bounded in order to guarantee integrability of the density of the constructed circular membrane.

\section{Examples}

\subsection{Regular polygons mapped to circle}

Let $\Omega$ be the regular $N$-sided polygon that inscribes the circle of radius 1 . In this case, the vertices are $w_{k}=\exp \left(\mathrm{i} \frac{2 k \pi}{N}\right)$ and the interior angles are $2 \pi / N$ yielding $\alpha_{k}=2 / N$. Fixing the prevertices so that $z_{k}=w_{k}$, the unit disc $\mathcal{D}$ is mapped to the $N$-sided polygon $\Omega$ by the Schwarz-Christoffel mapping:

$$
w=\int_{0}^{z} \frac{\mathrm{d} s}{\left(1-s^{N}\right)^{2 N}},
$$

cf Kober [13]. The density of the inhomogeneous membrane associated with the disc is

$$
\rho(z)=\rho_{0}\left|\frac{\mathrm{d} w}{\mathrm{~d} z}\right|^{2}=\rho_{0}\left|1-z^{N}\right|^{-4 N} .
$$

Figure 3 shows the homogeneous polygon inscribed in a unit circle and its corresponding inhomogeneous density for the case $N=8$. This density has eight singularities-one at each of the prevertices $z_{k}$ corresponding to each vertex of the polygon. Since $\beta_{k}=1-\alpha_{k}=$ $(N-2) / N<1$, the constructed density is in $L^{1}$. This means that the eigenfunctions are in $L^{2}$, [25]. Thus there is a circular inhomogeneous membrane that is isospectral to a regular polygon. Cureton and Kuttler [5] and Molinari [18] use this circular inhomogeneous membrane to compute the eigenvalues of the Laplacian over regular polygons for vastly different applications. 

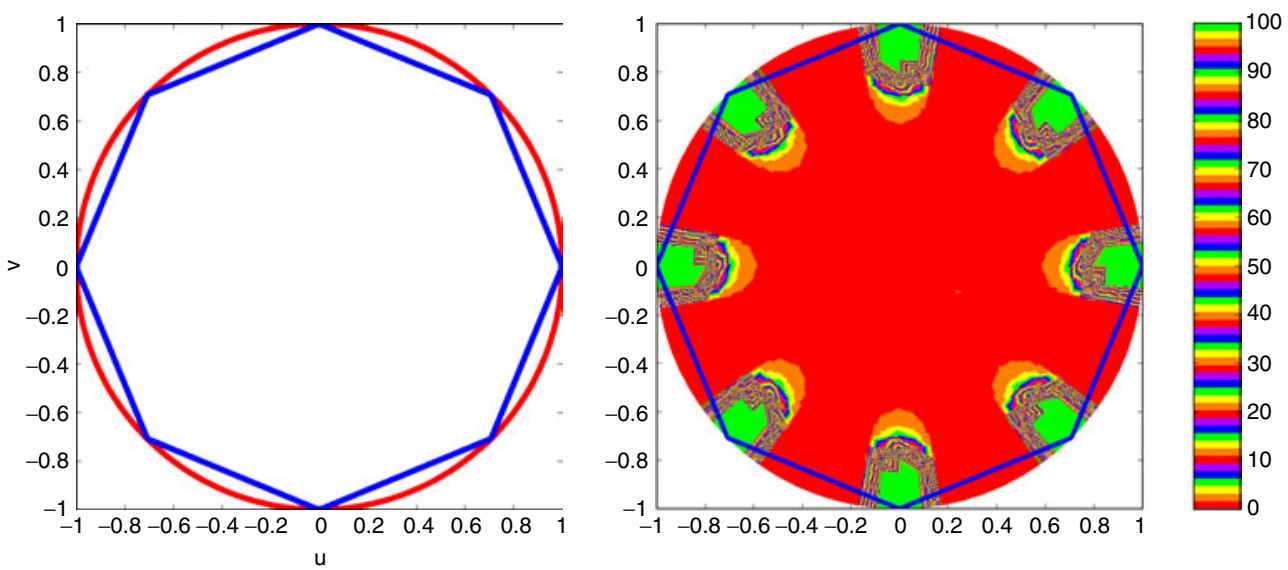

Figure 3. $N=8$, an eight-sided homogeneous polygon inscribed in a unit circle and its corresponding inhomogeneous density.
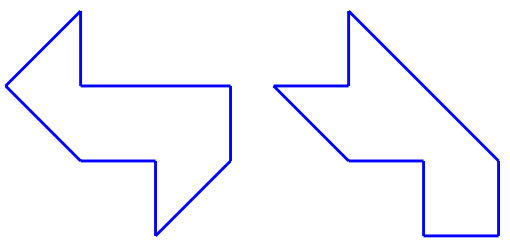

Figure 4. $\Omega_{1}$ bilby and $\Omega_{2}$ hawk.

\subsection{Gordon, Webb and Wolpert's shapes mapped to circle}

Let $\Omega_{1}$ and $\Omega_{2}$, shown in figure 4 , be the isospectral shapes discovered by Gordon, Webb and Wolpert $[4,3,9]$. These particular shapes were constructed using a right isosceles triangle and a series of reflections prescribed by two equivalent Cayley graphs [9]. Other triangles can be used to form similar pairs of isospectral shapes. These particular shapes were nicknamed bilby and hawk in [7].

The isospectrality of these shapes means that for homogeneous membranes with constant density $\rho_{0}$, the boundary value problems

$$
-\Delta u=\lambda \rho_{0} u \quad \text { in } \Omega_{i} \quad u=0 \quad \text { on } \partial \Omega_{i}
$$

have the same eigenvalues for $\Omega_{1}$ and $\Omega_{2}$. Using Schwarz-Christoffel mappings, we transform the unit discs $\mathcal{D}_{1}$ and $\mathcal{D}_{2}$ to $\Omega_{1}$ and $\Omega_{2}$ respectively. Prevertices for these maps were computed using the SC toolbox [8], and are shown for $\Omega_{1}$ and $\Omega_{2}$ in figure 5. Although the prevertices appear to cluster, they are in fact distinct because $F$ is one-to-one, $w_{k}=F\left(z_{k}\right)$ and the polygon vertices $w_{k}$ are distinct.

Consider the inhomogeneous membrane associated with each disc. Each has a density of the form

$$
\rho(z)=\rho_{0}\left|F^{\prime}(z)\right|^{2}=\rho_{0}|A|^{2} \prod_{k=1}^{n}\left|1-\frac{z}{z_{k}}\right|^{-2 \beta_{k}} .
$$

The values of $\alpha_{k}$ and $\beta_{k}$ for each of the polygons $\Omega_{1}$ and $\Omega_{2}$ are given in table 1 . These densities each have six singularities - one at each of the prevertices $z_{k}$ corresponding to a vertex whose interior angle $\alpha_{k} \pi$ is less than $\pi$. All of the $\beta_{k}$ satisfy $\beta_{k}<1$ and so each of the 

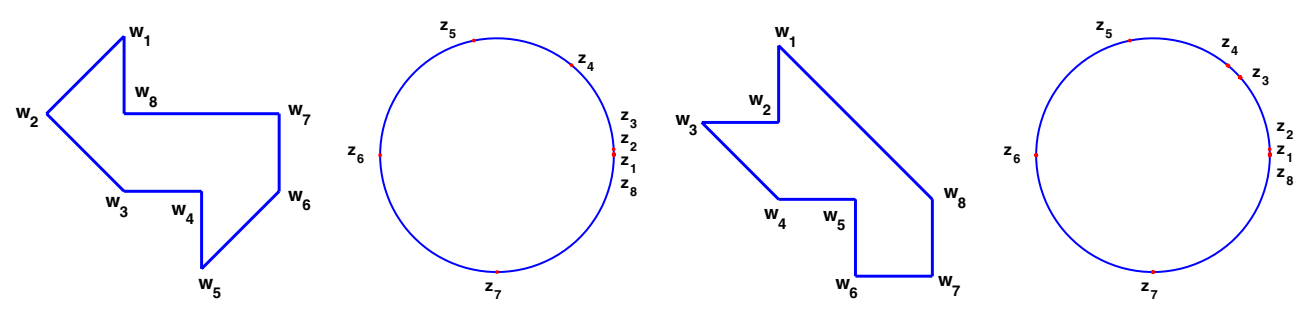

Figure 5. $\Omega_{1}, \mathcal{D}_{1}, \Omega_{2}$ and $\mathcal{D}_{2}$, showing vertices and prevertices.
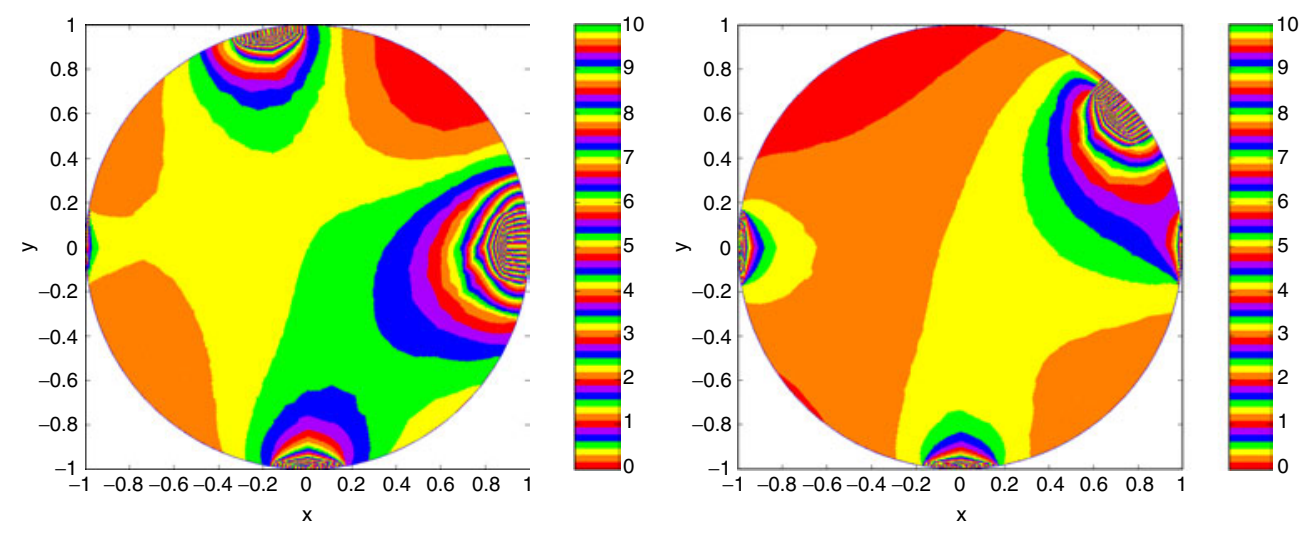

Figure 6. Gordon, Webb and Wolpert density for $\mathcal{D}_{1}$ and $\mathcal{D}_{2}$.

Table 1. Angle information.

\begin{tabular}{rrrrrrrrrr}
\hline$\Omega_{1}$ & $\alpha_{k}$ & $1 / 4$ & $1 / 2$ & $3 / 4$ & $3 / 2$ & $1 / 4$ & $3 / 4$ & $1 / 2$ & $3 / 2$ \\
& $\beta_{k}$ & $3 / 4$ & $1 / 2$ & $1 / 4$ & $-1 / 2$ & $3 / 4$ & $1 / 4$ & $1 / 2$ & $-1 / 2$ \\
& & & & & & & & & \\
$\Omega_{1}$ & $\alpha_{k}$ & $1 / 4$ & $3 / 2$ & $1 / 4$ & $3 / 4$ & $3 / 2$ & $1 / 2$ & $1 / 2$ & $3 / 4$ \\
& $\beta_{k}$ & $3 / 4$ & $-1 / 2$ & $3 / 4$ & $1 / 4$ & $-1 / 2$ & $1 / 2$ & $1 / 2$ & $1 / 4$ \\
\hline
\end{tabular}

constructed densities is in $L^{1}$ with eigenfunctions in $L^{2}$. Thus there are physically meaningful densities for circular inhomogeneous membranes that are isospectral to each other and to the homogeneous shapes constructed by Gordon, Webb and Wolpert. These densities are shown in figure 6 .

Isospectrality of the bilby and hawk shapes also holds for Neumann boundary conditions $[4,3]$ and has been verified numerically by Driscoll and Gottlieb [7]. Analysis identical to that used above shows that, in the case of Neumann boundary conditions, inhomogeneous circular membranes can be constructed that are isospectral to homogeneous membranes with the bilby and hawk shapes.

\section{Acknowledgments}

Both authors gratefully acknowledge partial support from the National Science Foundation. The second author also gratefully acknowledges the partial support of the Kentucky EPSCoR program. 


\section{References}

[1] Ahlfors L V 1979 Complex Analysis 3rd edn (New York: McGraw-Hill)

[2] Binns K J and Lawrenson P J 1963 Analysis and Computation of Electric and Magnetic Field Problems (New York: Pergamon)

[3] Webb D L, Gordon C and Wolpert S 1992 Isospectral plane domains and surfaces via Riemannian orbifolds Inventiones mathematicae 110 1-22

[4] Webb D L, Gordon C and Wolpert S 1992 One cannot hear the shape of a drum Bull. Am. Math. Soc. 27 134-8

[5] Cureton L M and Kuttler J R 1999 Eigenvalues of the Laplacian on regular polygons and polygons resulting from their disection J. Sound Vib. 220 83-98

[6] Driscoll T A 1996 A MATLAB Toolbox for Schwarz-Christoffel Mapping ACM Trans. Math. Softw. 22 168-86

[7] Driscoll T A and Gottlieb H P W 2003 Isospectral shapes with Neumann and alternating boundary conditions Phys. Rev. E 68 016702-1-6

[8] Driscoll T A and Trefethen L N 2002 Schwarz-Christoffel Mapping (Cambridge: Cambridge University Press)

[9] Gordon C and Webb D L 1996 You can't hear the shape of a drum Am. Sci. 84 46-55

[10] Gottlieb H P W 1988 Density distribution for isospectral circular membranes SIAM J. Appl. Math 48 948-51

[11] Gottlieb H P W 2004 Isospectral circular membranes Inverse Problems 20 155-61

[12] Kac M 1966 Can one hear the shape of a drum? Am. Math. Monthly 73 1-23

[13] Kober H 1957 Dictionary of Conformal Representations 2nd edn (New York: Dover)

[14] Kuttler J R and Sigillito V G 1984 Eigenvalues of the Laplacian in two dimensions SIAM Rev. 26 163-93

[15] Lamb H 1945 Hydrodynamics (New York: Dover)

[16] Laura P A A, Ercoli L, Grossi R O, Nagaya K and Sanchez-Sarmiento G 1985 Transverse vibrations of composite membranes of arbitrary boundary shape J. Sound Vib. 101 299-306

[17] Mazumdar J 1975 A review of approximate methods for determining the vibrational modes of membranes Shock Vib. Dig. 7 75-88

[18] Molinari L 1997 On the ground state of regular polygonal billiards J. Phys. A: Math. Gen. 30 6517-24

[19] Rudin W 1987 Real and Complex Analysis (New York: McGraw-Hill)

[20] Saff E B and Snider A D 1976 Fundamentals of Complex Analysis for Mathematics, Science and Engineering (Englewood Cliffs, NJ: Prentice-Hall)

[21] Schinzinger R and Laura P A A 1991 Conformal Mappings: Methods and Applications (Amsterdam: Elsevier)

[22] Sunada T 1985 Riemannian coverings and isospectral manifolds Ann. Math. 121 248-77

[23] Trefethen L N 1980 Numerical computation for the Schwarz-Christoffel transformation SIAM J. Sci. Stat. Comput. 1 82-108

[24] Trefethen L N and Driscoll T A 1998 Schwarz-Christoffel mapping in the computer era Proc. Int. Congress of Mathematicians vol 3 (Berlin: Springer) pp 533-42

[25] Weinberger H 1974 Variational Methods for Eigenvalue Approximation (Philadelphia, PA: SIAM) 